\title{
ESTUDO DO COMPORTAMENTO FLUIDODINÂMICO DA BIOADSORÇÃO EM LEITO FIXO DE ÍONS CÁDMIO EM RESÍDUO DA EXTRAÇÃO DE ALGINATO DE ALGA MARROM
}

\author{
G. F. LOPES ${ }^{1}$, E. NISHIKAWA ${ }^{1}$, M.G. A. VIEIRA ${ }^{1}$, M. G. C. SILVA ${ }^{1}$ \\ ${ }^{1}$ Universidade Estadual de Campinas, Faculdade de Engenharia Química, Departamento de \\ Desenvolvimento de Processo e Produtos \\ E-mail para contato: meuris@ feq.unicamp.br
}

\begin{abstract}
RESUMO -A busca constante por tratamentos de remoção de metais tóxicos de efluentes vem estimulando o estudo da bioadsorção pela versatilidade e eficiência em baixas concentrações. Neste trabalho, avaliou-se o uso do resíduo da extração de alginato como bioadsorvente de cádmio em sistema dinâmico de leito fixo, visando agregar valor a um resíduo gerado na produção do alginato. $\mathrm{O}$ estudo do comportamento fluidodinâmico indicou que a vazão de $0,5 \mathrm{~mL} \cdot \mathrm{min}^{-1}$ resulta na maior eficiência de operação em leito fixo adsortivo. Estudos anteriores revelaram a alta rugosidade do bioadsorvente, além de evidenciar a presença dos biopolímeros alginato e fucoidana, considerados fundamentais no processo de bioadsorção de metais.
\end{abstract}

\section{INTRODUÇÃO}

O aumento da rigidez das leis ambientais nos últimos anos fomenta pesquisas de tratamento de efluentes que sejam mais eficazes e baratos. $\mathrm{O}$ tratamento de resíduos contendo metais tóxicosse destaca devido à sua alta toxicidade e ampliação biológica. Dentre tais metais, o cádmio é largamente utilizado como componente na formulação de pigmentos, bateria, na estabilização de plásticos (policloreto de vinila) e pela indústria de mineração(Prasad e Hagemeyer, 2013; Prasad, 1995). É também altamente tóxico, sendo que em humanos pode causar disfunção renal e do fígado(Cetesb, 2012), além de fragilidade óssea. Nas plantas, o cádmio prejudica o crescimento e a produção de clorofila(Daset al., 1997).

As principais técnicas de remoção de metais tóxicos, como precipitação química, filtração e osmose reversa, apresentam limitações(El-Bayaaet al., 2009). Por esse motivo, diferentes métodos, como a bioadsorção, vêm ganhando atenção pela sua versatilidade, economia (a depender do adsorvente) e eficiência na remoção principalmente a baixas concentrações do metal (Bhattacharyaet al., 2006).

Dessa forma, a motivação deste trabalho é utilizar um bioadsorvente de baixo custo que supra as exigências legislativas. O material estudado foi o resíduo da extração de alginato da alga Sargassum filipendula, tendo em vista que o alginato é amplamente utilizado pela 
indústria e o resíduo ainda é pouco estudado. O bioadsorvente foi empregado em coluna de leito fixo e parâmetros de eficiência e de transferência de massa foram determinados para diferentes vazões de operação.

\section{MATERIAIS E METODOLOGIA}

\subsection{Preparo do Bioadsorvente}

A alga marrom Sargassum filipendula, coletada na Praia das Cigarras - São Sebastião, SP,foi lavada, triturada e peneirada (aberturas de 16 e $32 \mathrm{Mesh}$ ) para o processo de extração.O biomaterial foi armazenado abaixo de $-10^{\circ} \mathrm{C}$ para evitar o crescimento de fungos.

A extração do alginato foi realizada utilizando o método de McHugh (1987), no qual $15 \mathrm{~g}$ de alga foi posta em contato com $500 \mathrm{~mL}$ de formaldeído a $0,4 \%$ por 30 minutos. A alga foi lavada com água deionizada e mantida em contato com $500 \mathrm{~mL}$ de ácido clorídrico sob agitação constante por $2 \mathrm{~h}$. Após nova lavagem com água deionizada,foram adicionados 350 $\mathrm{mL}$ de carbonato de sódio $2 \%$ sob agitação constante por $5 \mathrm{~h}$ a $60^{\circ} \mathrm{C}$. A mistura foi filtrada a vácuo, sendo o resíduo lavado exaustivamente com água deionizada e seco em estufa a $60^{\circ} \mathrm{C}$ por $24 \mathrm{~h}$. Após essa etapa, o resíduo foi triturado e peneirado para sua utilização como bioadsorvente. O diâmetro médio das partículas foi de $0,737 \mathrm{~mm}$.Devido à expansão do bioadsorvente no interior da coluna pela absorção de água, o material é hidratado por $12 \mathrm{~h}$ para que seu volume não varie no decorrer do processo.

\subsection{Ensaio de Adsorção em Leito Fixo}

Os ensaios foram realizados com uma coluna de vidro de $0,7 \mathrm{~cm}$ de diâmetro interno e 7 $\mathrm{cm}$ de altura sob fluxo ascendente a fim de evitar caminhos preferenciais e empacotamento do leito(Lima, 2016). Para o adsorvato, utilizou-se uma solução de $1 \mathrm{mmol} . \mathrm{L}^{-1}$ de nitrato de cádmio em pH 3,5 de modo a evitar a precipitação química do metal. As alíquotas foram retiradas por um coletor de fração automático (FC 203B Fraction Collector) em intervalos pré-definidos e a concentração de cádmio foi obtida pelo espectrofotômetro de absorção atômica (Perkin Elmer, modelo Analyst 100).

Para o estudo do comportamento fluidodinâmico, três vazões diferentes foram avaliadas: 0,5; 0,8 e 1,0 mL.min ${ }^{-1}$, a fim de determinar a vazão mais adequada de acordo com parâmetros de eficiência na remoção e de transferência de massa. O aumento da vazão diminui a resistência das fases e,por consequência, a Zona de Transferência de Massa (ZTM). Contudo, a diminuição no tempo de contato da amostra com o adsorvente dificulta a adsorção (Nishikawa et al., 2012), fazendo-se necessário um estudo detalhado da vazão do efluente.

O cálculo da ZTM pode ser feito através de um método simplificado, expresso através da Equação 1(Geankoplis, 1993).

$$
\mathrm{ZTM}=\left(1-\frac{\mathrm{q}_{\mathrm{u}}}{\mathrm{q}}\right) * \mathrm{H}_{\text {total }}
$$

em que $\mathrm{H}_{\text {total }}$ é a altura da coluna $(\mathrm{cm})$; $\mathrm{q}_{\mathrm{u}}$ é a quantidade adsorvida por unidade de adsorvente no ponto de ruptura, definido como a condição em que a concentração de saída é $5 \%$ da 
concentração de entrada; q é a quantidade adsorvida por massa de adsorvente no ponto de saturação, onde a concentração de saída é $95 \%$ da concentração de entrada. Obtém-se qu e q a partir das Equações 2 e 3, respectivamente.

$$
\begin{aligned}
& \mathrm{q}_{\mathrm{u}}=\frac{\mathrm{C}_{0} * \mathrm{Q}}{\mathrm{m}} \int_{0}^{\mathrm{t}_{\mathrm{b}}}\left(1-\frac{\mathrm{C}(\mathrm{t})}{\mathrm{C}_{0}}\right) \mathrm{dt} \\
& \mathrm{q}=\frac{\mathrm{C}_{0} * \mathrm{Q}}{\mathrm{m}} \int_{0}^{\mathrm{t}_{\mathrm{t}}}\left(1-\frac{\mathrm{C}(\mathrm{t})}{\mathrm{C}_{0}}\right) \mathrm{dt}
\end{aligned}
$$

naqual $t_{b}$ é o tempo de ruptura, $t_{t} o$ tempo em que a capacidade total da coluna foi atingida, $\mathrm{Q}$ a vazão do sistema, $\mathrm{m}$ a massa seca de resíduo e $\mathrm{C}_{0}$ a concentração inicial.Outros parâmetros úteis no scale-up da coluna podem ser calculados. O tempo tzrequerido para que a ZTM percorra o equivalente ao seu comprimento e o tempote de saída da ZTM do leito estão apresentados nas Equações 4 e 5, respectivamente.

$$
\begin{aligned}
& \mathrm{t}_{\mathrm{z}}=\frac{\mathrm{V}_{\mathrm{E}}-\mathrm{V}_{\mathrm{B}}}{\mathrm{Q}_{\mathrm{W}}} \\
& \mathrm{t}_{E}=\frac{\mathrm{V}_{\mathrm{E}}-\mathrm{V}_{\mathrm{B}}}{Q}
\end{aligned}
$$

em que $V_{B}$ é o volume da solução tratada até o ponto de exaustão (L); $V_{E}$ é o volume tratado até o ponto de exaustão (L) e Q é a vazão da solução $\left(L \cdot h^{-1}\right)$.O parâmetro $F$ é a fraçãodo adsorvente capaz de adsorver metal na ZTM, o tempo de formação inicial desta é $\mathrm{t}_{\mathrm{f}}(\mathrm{h})$, e a sua velocidade é $U_{z}\left(\mathrm{~cm} \cdot \mathrm{h}^{-1}\right)$. Tais parâmetros são apresentados nasEquações 6,7 e 8 , respectivamente. Por fim, a porcentagem de remoção no leito \%RL foi calculada utilizando a quantidade de metal total em solução até a ruptura e saturação da coluna.

$$
\begin{aligned}
& \mathrm{F}=\frac{\int_{\mathrm{V}_{\mathrm{B}}}^{\mathrm{V}_{\mathrm{E}}}\left(\mathrm{C}_{0}-\mathrm{C}\right) \mathrm{dV}}{\mathrm{C}_{0}\left(\mathrm{~V}_{\mathrm{E}}-\mathrm{V}_{\mathrm{B}}\right)} \\
& \mathrm{t}_{\mathrm{f}}=(1-\mathrm{F}) \mathrm{t}_{\mathrm{z}} \\
& \mathrm{U}_{\mathrm{z}}=\frac{\mathrm{h}}{\mathrm{t}_{\mathrm{E}}-\mathrm{t}_{\mathrm{f}}}
\end{aligned}
$$

\section{RESULTADOS E DISCUSSÃO}

A Figura 1 apresenta as curvas de rupturas para as vazões 0,5, 0,8 e 1,0 mL.min ${ }^{-1}$. Os valores de ZTM, qu, q, tz, te, tf, F, UZ, \%RL ruptura, \%RL totalestão listados na Tabela 1.

A curva de ruptura para a vazão de $0,5 \mathrm{~mL} \cdot \mathrm{min}^{-1}$ resulta na maior porcentagem de remoção no ponto de ruptura $(89,45 \%)$ e quando saturada $(44,99 \%)$ e menor ZTM $(2,56 \mathrm{~cm})$, indicando uma menor resistência à transferência de massa.A maiorremoção pode ser decorrente do maior tempo de residência do efluente na coluna, onde o bioadsorvente teria contato com a solução contaminada por tempo suficiente para a adsorção.

Os parâmetros tz e $U_{z}$ não apresentaram uma tendência definida com relação à vazão. Entretanto, esperava-se uma redução em tz e um aumento em $U_{z}$ com o aumento da vazão, 
uma vez que estão relacionados com a movimentação da ZTM no leito. Com relação a tE e F, houve aumento destes acompanhando a vazão, enquanto $t_{f}$ apresentou redução ao aumentar a vazão de alimentação. $\mathrm{O}$ valor de $\mathrm{F}$ representa a fração de adsorvente ainda capaz de adsorver no ponto de ruptura (Nidheesh et al., 2012), assim, quanto menor seu valor mais eficiente é o uso da coluna. Verificou-se que para a vazão de $0,5 \mathrm{~mL} \cdot \mathrm{min}^{-1}$, a fração $\mathrm{F}$ resultou no menor valor. Considerando-se os parâmetros calculados, determinou-se que a vazão de $0,5 \mathrm{~mL} \cdot \mathrm{min}^{-1}$ é a mais adequada à operação, pois resulta em menor resistência à transferência de massa.

Figura 1 -Comportamento fluidodinâmico da bioadsorção de cádmio em resíduo da extração de alginato.

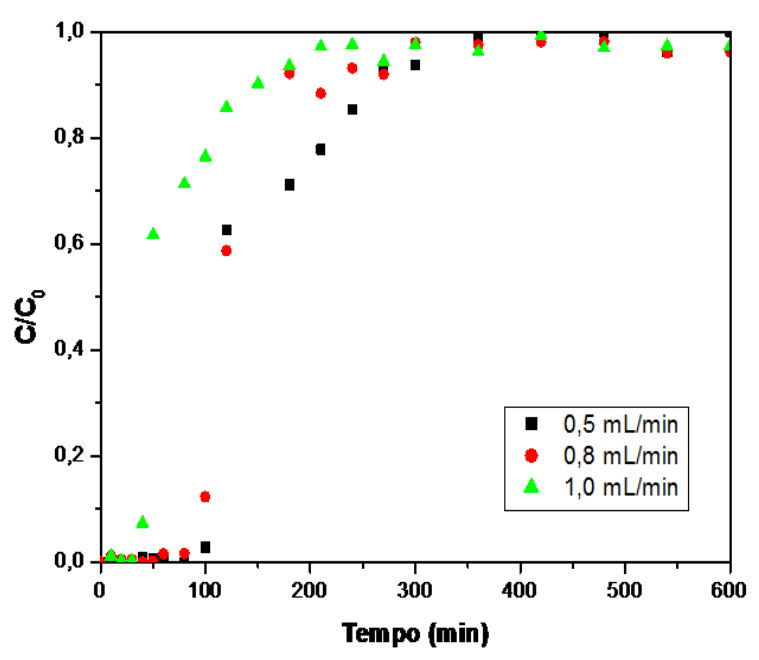

Tabela 1 - Parâmetros do estudo fluidodinâmico.

\begin{tabular}{|c|c|c|c|}
\hline Vazão (mL.min $\left.{ }^{-1}\right)$ & 0,5 & 0,8 & 1,0 \\
\hline ZTM (cm) & 2,56 & 2,76 & 4,16 \\
\hline $\mathrm{q}_{\mathrm{u}}\left(\mathrm{mmol} \cdot \mathrm{g}^{-1}\right)$ & 0,1401 & 0,2278 & 0,1001 \\
\hline $\mathrm{q}\left(\mathrm{mmol} . \mathrm{g}^{-1}\right)$ & 0,2210 & 0,3757 & 0,2467 \\
\hline $\mathrm{tz}_{\mathrm{z}}(\mathrm{h})$ & 3,59 & 3,32 & 3,99 \\
\hline$t_{E}(h)$ & 5,27 & 4,76 & 4,60 \\
\hline $\mathrm{F}$ & 0,3660 & 0,3938 & 0,5941 \\
\hline $\mathrm{t}_{\mathrm{f}}(\mathrm{h})$ & 2,27 & 2,01 & 1,62 \\
\hline $\mathrm{U}_{\mathrm{Z}}\left(\mathrm{cm} \cdot \mathrm{min}^{-1}\right)$ & 2,34 & 2,55 & 2,35 \\
\hline$\% \mathrm{RL}_{\text {ruptura }}$ & 89,45 & 87,50 & 71,94 \\
\hline$\% \mathrm{RL}_{\text {total }}$ & 44,99 & 43,66 & 36,09 \\
\hline
\end{tabular}

Costa et al. (2016) caracterizaram o bioadsorvente utilizado neste trabalho em publicação anterior. O observado foi que a superfície do bioadsorvente é bastante rugosa e também é destacada a presença de diatomáceas, proveniente da alga in natura e que não foram totalmente removidas na extração de alginato. 
Ainda nesse estudo foi notado que a composição do resíduo apresenta significativa de sódio provavelmente devido ao processo de extração de alginato, onde se utilizou carbonato de sódio.

Os autores verificaram em espectros de FT-IR as bandas em 3287, 1607 e $1537 \mathrm{~cm}^{-1}$, características de estiramentos $\mathrm{OH}, \mathrm{CH}$ de grupos carboxílicos e grupos amino de proteínas, respectivamente (Sheng et al., 2004; Kleinübing et al., 2013). As bandas em 1237 e $808 \mathrm{~cm}^{-1}$ indicam a presença do biopolímero fucoidana, que segundo Fourest e Volesky (1996), tem papel relevante na bioadsorção de metais tóxicos. Entretanto, o maior responsável pela capacidade de remoção de íons metálicos é o alginato, e as bandas em 1032 e $1052 \mathrm{~cm}^{-1}$ evidenciam que mesmo após o processo de extração, ainda resta alginato no resíduo. Assim, o resíduo ainda possui grupos funcionais para a bioadsorção de metais tóxicos, o que corrobora com os resultados promissores de remoção obtidos no presente trabalho.

\section{CONCLUSÕES}

No estudo fluidodinâmico verificou-se que a vazão de $0,5 \mathrm{~mL} \cdot \mathrm{min}^{-1}$ é a mais adequada para a operação do ponto de vista de transferência de massa. As porcentagens de remoção até o ponto de ruptura e de saturação foram de $89,45 \%$ e $44,99 \%$, respectivamente, e a quantidade total de cádmio adsorvida por grama de adsorvente foi de $0,221 \mathrm{mmol} . \mathrm{g}^{-1}$.A partir dos resultados, pode-se concluir que o resíduo da extração de alginato da alga Sargassum filipendulaé um material promissor para a remoção de metais tóxicos como o cádmio de efluentes industriais.

\section{AGRADECIMENTOS}

Os autores agradecem ao PIBIC/CNPq e à Fapesp(2014/04050-5) pelo apoio financeiro para realizaçãodeste trabalho.

\section{REFERÊNCIAS}

BHATTACHARYA, A.; MANDAL, S.; DASA, S. Adsorption of Zn(II) from aqueous solution by using different adsorbents. Chem Eng J, v. 123, p. 43-51, 2006.

CETESB. Cádmio e seus compostos. Disponível em: <http://cetesb.sp.gov.br/wpcontent/uploads/sites/47/2013/11/cadmio.pdf>. Acesso em: 27 dez. 2016.

COSTA, C. S. D.; CARDOSO, S. L.; NISHIKAWA, E.; VIEIRA, M. G. A.; SILVA, M. G. C. Characterization of the Residue from Double Alginate Extraction from Sargassum filipendula Seaweed. Chem Engineer Trans, v. 52, p. 133-138, 2016.

DAS, P.; SAMANTARAY, S.; ROUT, G. R. Studies On Cadmium Toxicity In Plants: A Review. Environ Pollut, v. 98, p. 29-36, 1997.

EL-BAYAA, A. A.; BADAWY, N. A.; ALKHALIK, E. Abd. Effect of ionic strength on the adsorption of copper and chromium ions by vermiculite pure clay mineral.J Hazard Mater, v. 170, n. 2-3, p. 1204-1209, 2009. 
FOUREST, E.; VOLESKY, B. Contribution of Sulfonate Groups and Alginate to Heavy Metal Biosorption by the Dry Biomass of Sargassum fluitans. Environ Sci and Technol, v. 30, p. 277-282, 1996.

GEANKOPLIS, C. J. Transport Processes and Unit Operations. In: 3. ed., Prentice-Hall International, p. 702-704, 1993.

KLEINUBING, S. J.; GAIA, F.; BERTAGNOLlI, C.; SILVA, M. G. C. Extraction of alginate biopolymer present in marine alga Sargassum filipendula and bioadsorption of metallic ions. Mat Res, v. 16, n. 2, p. 481-488, 2013.

LIMA, L. Adsorção de hidrocarbonetos btx por argila organofílica em sistema dinâmico de leito fixo (Dissertação). Universidade Estadual de Campinas, 2016.

MCHUGH, D.J. Production, properties and uses of Alginates. FAO Fisheries Technical Papers, 1987, p. 58-115.

NIDHEESH, P. V.; GANDHIMATHI, R.; RAMESH, S. T.; SINGH, T. S. A. Adsorption and desorption characteristics of crystal violet in bottom ash column.JUEE, v. 6, n. 1, p 1829, 2012.

NISHIKAWA, E.; ALMEIDA NETO, A. F.; SILVA, M. G. C. da; VIEIRA, M. G. A.; Estudos de Transferência de massa de zinco em leito poroso de vermiculita expandida, 01/2012, COBEQ2012 - XIX Congresso Brasileiro de Engenharia Química,Vol. 1, pp.1122-1131, Búzios, RJ, Brasil, 2012

PRASAD, M.N.V.; HAGEMEYER, Jürgen.Heavy Metal Stress in Plants: From Molecules to Ecosystems.Springer Science \& Business Media, 2013.

PRASAD, M. N. V. Plants, Cadmium Toxicity And Tolerance In Vascular. Environ Exp Bot, v. 35, p. $525-545,1995$.

SHENG, P. X.; WEE, K. H.; TING, Y. P.; CHEN, J. P. Biosorption of copper by immobilized marine algal biomass. Chem Eng J, v. 136, p.156-163, 2008. 